\title{
A Comparative Study on the Effect of Doshahara Basti and Vaitarana Basti in the management of Amavata
}

\section{Research article}

\author{
Asharani DH ${ }^{1 *}$, Channabasavanna BM+2, Srinivasulu $\mathbf{M}^{3}$ \\ 1. PG Scholar, 2. Assistant professor, 3. HOD, Professor \\ Department of Panchakarma, NKJ Ayurvedic College, Bidar
}

\begin{abstract}
In present era $80 \%$ of the disorders in the world are of Samarogas. Out of them the most crippling disorder, which hampers the economy of the world as well as the individual, are musculo-skeletal disorders. These are best known as Rheumatic disorders. Out of them many disorders have got signs \& symptoms similar to Amavata. So the Amavata description given in the Madhavanidana covers a variety of Rheumatological disorders. Due to wide spectrum of disease, much prevalence in the society and lack of effective medicament, the disease is being chosen for the study. This research study was conducted on 30 patients with classical sign \& symptoms of Amavata by adopting proper assessment criteria. In group A 15 patients were treated with Prasarini Taila and Doshahara Basti for 8 consecutive days. In group B 15 patients were treated with Prasarini Taila and Vaitarana Basti for 8 consecutive days. The pharmacodynamic property of Basti dravyas are having Laghu-Tikshna guna, Katu-Tikta rasa, Ushna virya are against the Guru, Pichchila, Sheeta properties of Ama. Thus Basti proves an effective treatment in Amavata. The overall effect of therapy in Group A was $78.57 \%$ and in Group B was $51.19 \%$.
\end{abstract}

Key words: Amavata, Rheumatic disorders, Vaitarana basti, Doshahara Basti.

\section{Introduction}

Amavata is the common most crippling and disabling disorder in the world as well as in India. The word Amavata is self explanatory, Aama + Vata indicates the prime components of the disease. Since Aama is having equal gunas to kapha, its affinity is mostly towards shleshma sthanas, hence the sthanasamshraya of the disease is at shleshma sthanas i.e synovial joints, heart, lungs, muscles, fascia etc.

*Corresponding Author:

\section{Asha Rani DH}

PG scholar,

Department of Panchakarma,

NKJ Ayurvedic College, Bidar.
In light of modern science, Amavata word has been used extensively with comparison to rheumatoid arthritis, rheumatic arthritis and many more rheumatological disorders.(1)

In present busy and mechanical life, one can't follow the rules of 'Dinacharya' and 'Ritucharya' described in 'Ayurveda'. Every person indulging in improper food and habits which are not fit to the constitution of the body. Such food which have very less nutritional values and similar properties to Viruddhahara causes provocation of Vata dosha and Agni dusti, by these prime factors a primordial disease manifesting factor developed known as AAMA.

The disease was first explained in detail manner by Acharya Madhavakara in 
Madhava nidhana(2) during the period of $7^{\text {th }}$ century $\mathrm{AD}$. He covers a veriety of Rheumatological disorders under the light of Amavata. It is a disorder characterized by Ama dosha, Vata dosha, Kapha dosha morbidly. This is a disease where in Rasavaha srotas is primarily involved. Because of this, the pain spreads from one joint to another joint very quickly. The line of treatment described for the disease as "Langhanam Swedanam Tiktha (3)" can be summarized under following captions.

1) Measures to bring Agni to normal state.

2) Measure to digest Aama.

3) Measures to eliminate vitiated Vata and Aama.

Aims \& objectives:

1) To study role of basti Chikitsa in Amavata in detail.

2) To assess the efficacy of Doshahara basti in Amavata.

\section{Materials and Method}

This research study was conducted on 30 patients with classical sign \& symptoms of Amavata by adopting proper assessment criteria.

Group A: 15 patients were treated with Prasarini Taila(4) and Doshahara Basti(5) for 8 consecutive days.

Group B: 15 patients were treated with Prasarini Taila and Vaitarana Basti(6) for 8 consecutive days.

\section{Source of Data}

For this study the patients were selected from the IPD \&OPD of Panchakarma department of Sri Siddharoodh Charitable hospital attached to N.K.J.A.M.C \& P. G. CENTRE \& a case proforma was prepared by incorporating the signs \& symptoms of katigraha.

\section{Study Design}

The study design was randomized. Patients were selected according to the inclusion and exclusion criteria.

\section{Criteria for selection.}

a) Inclusion criteria:-

1. Patients of age group between 20 to 60 years irrespective of sex.

2. Patients presenting with signs and symptoms of Amavata mentioned in classical texts.

3. Patients fit for Basti Chikitsa

b) Exclusion criteria

1. Patients with systemic pathologies like cardiac diseases, renal diseases, SLE etc.

2. Patients with tuberculosis of spine, spinal tumours, vertebral fractures, connective tissue disorders \& surgical conditions.

3. Chronic patients like swan neck and ulnar deformity.

4. All the conditions where basti is contraindicated.

\section{Assessment Criteria}

Subjective parameters

- Shula

- Sthambha

- Graha

- Shotha

- Vaivarnata

\section{Objective parameters}

- ESR

- ASO

Chart Showing the Grading of Parameters

\section{1) Shula}

\begin{tabular}{|l|l|}
\hline No pain & Grade 0 \\
\hline Mild pain & Grade 1 \\
\hline Moderate pain & Grade 2 \\
\hline Severe pain & Grade 3 \\
\hline Most excruciating pain & Grade 4 \\
\hline
\end{tabular}

\section{2) Sthamba}

\begin{tabular}{|l|l|}
\hline $\begin{array}{l}\text { No stiffness or stiffness } \\
\text { lasting for 5 minutes }\end{array}$ & Grade 0 \\
\hline $\begin{array}{l}\text { Stiffness lasting for 6 minutes } \\
\text { to } 30 \text { minutes }\end{array}$ & Grade 1 \\
\hline $\begin{array}{l}\text { Stiffness lasting for 35 } \\
\text { minutes to } 1 \text { hour }\end{array}$ & Grade 2 \\
\hline
\end{tabular}




\begin{tabular}{|l|l|}
\hline $\begin{array}{l}\text { Stiffness lasting for } 1 \frac{1}{2} 2 \text { hour } \\
\text { to } 2 \text { hour }\end{array}$ & Grade 3 \\
\hline $\begin{array}{l}\text { Stiffness lasting for more than } \\
2 \text { hour }\end{array}$ & Grade 4 \\
\hline
\end{tabular}

\section{3) Graha}

\begin{tabular}{|l|l|}
\hline Normal joint motion & Grade 0 \\
\hline About 25 - 49\% loss of motion & Grade 1 \\
\hline About 50\% loss of motion & Grade 2 \\
\hline About 75\% loss of motion & Grade 3 \\
\hline $\begin{array}{l}100 \% \text { loss of motion or } \\
\text { complete ankyloses of the } \\
\text { joint }\end{array}$ & Grade 4 \\
\hline
\end{tabular}

\section{4) Shotha}

\begin{tabular}{|l|l|}
\hline No Swelling & Grade 0 \\
\hline $\begin{array}{l}\text { Joint swelling which may not } \\
\text { be apparent on casual } \\
\text { inspection, but difficult to } \\
\text { recognize on casual } \\
\text { observation }\end{array}$ & \\
\hline $\begin{array}{l}\text { Joint swelling obvious even } \\
\text { on casual observation }\end{array}$ & Grade 2 \\
\hline Markedly abnormal swelling & Grade 3 \\
\hline $\begin{array}{l}\text { Joint swelling to a maximally } \\
\text { abnormal degree }\end{array}$ & Grade 4 \\
\hline
\end{tabular}

\section{5) Vaivarnata}

\begin{tabular}{|l|l|}
\hline Absent & Grade 0 \\
\hline Mild & Grade 1 \\
\hline Mooderate & Grade 2 \\
\hline Markked & Grade 3 \\
\hline Severe & Grade 4 \\
\hline
\end{tabular}

\section{6) ESR}

\begin{tabular}{|l|l|}
\hline Oto20mm & Grade 0 \\
\hline 21 to 40 & Grade 1 \\
\hline 41 to60 & Grade 2 \\
\hline 61 to 80 & Grade 3 \\
\hline Above 80 & Grade 4 \\
\hline
\end{tabular}

\section{7) ASO}

\begin{tabular}{|l|l|}
\hline Normal $(<200 \mathrm{IU} / \mathrm{ml})$ & Grade 0 \\
\hline Low $(200-300 \mathrm{IU} / \mathrm{ml})$ & Grade 1 \\
\hline Average $(301-400 \mathrm{IU} / \mathrm{ml})$ & Grade 2 \\
\hline Moderate $(401-500 \mathrm{IU} / \mathrm{ml})$ & Grade 3 \\
\hline High $($ More than $501 \mathrm{IU} / \mathrm{ml})$ & Grade 4 \\
\hline
\end{tabular}

\section{Intervention Chart}

\section{Group A}

\begin{tabular}{|c|c|c|c|c|}
\hline $\begin{array}{l}\text { SI } \\
\text { No. }\end{array}$ & Procedure & Drug & Dose & Duration \\
\hline \multirow[t]{3}{*}{1.} & Poorva Karma & & & \\
\hline & a)Sarvanga Abhyanga & Murchita tila taila & Q.S & $30-40$ mins \\
\hline & b)Bhashpa sweda & & & $\begin{array}{l}\text { Till samyak swinna } \\
\text { lakshana appears. }\end{array}$ \\
\hline \multirow[t]{6}{*}{2.} & Pradhana Karma & & & \\
\hline & a)Anuvasana Basti & Prasarini taila & $\begin{array}{l}100 \mathrm{ml} \text { appro } \\
(2 \text { pala) }\end{array}$ & $1^{\text {st }}, 3^{\text {rd }}, 5^{\text {th }}, 7^{\text {th }}, 8^{\text {th }}$ day \\
\hline & \multirow[t]{4}{*}{ b)Niruha Basti } & Madhu & $50 \mathrm{gm}$ & \multirow{4}{*}{$2^{\text {nd }}, 4^{\text {th }}, 6^{\text {th }}$ day } \\
\hline & & Saindhava lavana & $12 \mathrm{gm}$ & \\
\hline & & Prasarini taila & $\begin{array}{l}80 \mathrm{ml}\left(1 / 5^{\text {th }} \text { of }\right. \\
\text { total niruha } \\
\text { matra })\end{array}$ & \\
\hline & & Shatahva kalka & $10 \mathrm{gm}$ & \\
\hline
\end{tabular}




\begin{tabular}{|l|l|l|l|l|}
\hline & & $\begin{array}{l}\text { Shatpuspha, } \\
\text { Vaca, Hingu, } \\
\text { Triphala, Rasana, } \\
\text { Devdaru } \\
\text { Kashaya }\end{array}$ & $400 \mathrm{ml}$ & \\
\hline 3. & Paschat Karma & $\begin{array}{l}\text { Total Appro } \\
\text { Lifting legs,padding } \\
\text { to the buttocks, anti } \\
\text { clockwise massage } \\
\text { to abdomen etc. }\end{array}$ & $550 \mathrm{ml}$ & $3-5$ mins \\
\hline
\end{tabular}

\section{Group B}

\begin{tabular}{|c|c|c|c|c|}
\hline $\begin{array}{l}\text { SI } \\
\text { No. }\end{array}$ & Procedure & Drug & Dose & Duration \\
\hline \multirow[t]{3}{*}{1.} & Poorva Karma & & & \\
\hline & a)Sarvanga Abhyanga & Murchita tila taila & Q.S & $30-40$ mins \\
\hline & b)Bhashpa sweda & & & $\begin{array}{l}\text { Till samyak swinna } \\
\text { lakshana appears. }\end{array}$ \\
\hline \multirow[t]{8}{*}{2.} & Pradhana Karma & & & \\
\hline & a)Anuvasana Basti & Prasarini taila & $\begin{array}{l}100 \mathrm{ml} \text { appro } \\
(3 \text { pala })\end{array}$ & $1^{\text {st }}, 3^{\text {rd }}, 5^{\text {th }}, 7^{\text {th }}, 8^{\text {th }}$ day \\
\hline & \multirow[t]{5}{*}{ b)Niruha Basti } & Guda & $50 \mathrm{gm}$ & \multirow{5}{*}{$2^{\text {nd }}, 4^{\text {th }}, 6^{\text {th }}$ day } \\
\hline & & Saindhava lavana & $12 \mathrm{gm}$ & \\
\hline & & Prasarini taila & $\begin{array}{l}80 \mathrm{ml}\left(1 / 5^{\text {th }} \text { of }\right. \\
\text { total niruha } \\
\text { matra) }\end{array}$ & \\
\hline & & Chincha kalka & $10 \mathrm{gm}$ & \\
\hline & & Gomutra & $400 \mathrm{ml}$ & \\
\hline & & Total Appro & $550 \mathrm{ml}$ & \\
\hline 3. & Paschat Karma & $\begin{array}{l}\text { Lifting legs,padding } \\
\text { to the buttocks, anti- } \\
\text { clockwise massage } \\
\text { to abdomen etc. }\end{array}$ & & $3-5$ mins \\
\hline
\end{tabular}

\section{Observation:}

Maximum patients registered in the age group of 41-50 years of age $56.67 \%$. Most of the patients were female $63 \%$ as compared to male patients $37 \%$. Out of 30 patients, $96.67 \%$ were Hindus, and $3.33 \%$ were Muslims. $93.33 \%$ patients were married. $6.67 \%$ were unmarried. $6.67 \%$ patients were uneducated, remaining $93.33 \%$ were distributed in different level like primary $26.67 \%$, secondary $30 \%$, graduate $23.33 \%$ and post graduate $13.33 \%$. Majority of the patients $43.33 \%$ in this series were belonging to middle economic status, while $23.33 \%$ were belonging to upper middle class.

Maximum number of patients $60 \%$ were possessing Vata-Kapha Prakruti, 23.33\% Vata-Pitta and $16.67 \%$ patients were of Pitta-Kapha Prakruti. All the patients were of Manda 
Agni 76.67\% and Visama Agni 23.33\% . Maximum patients 76.67\% had Madhyama Koshtha, which was followed by Krura Kostha $13.33 \%$ and Mrudu Kostha $10 \%$.

Results

Group A - subjective parameters:

\begin{tabular}{|c|c|c|c|c|c|c|c|c|c|}
\hline $\begin{array}{c}\text { Sr.N } \\
\text { o }\end{array}$ & $\begin{array}{l}\text { Sympto } \\
\mathrm{ms}\end{array}$ & $\begin{array}{l}\text { B.T } \\
\text { Mean } \pm S \\
\text { E }\end{array}$ & $\begin{array}{l}\text { Follow } \\
\text { up }\end{array}$ & $\begin{array}{l}\text { A.T } \\
\text { Mean } \pm S \\
. \mathrm{E}\end{array}$ & $\begin{array}{l}\text { d. } \\
\text { f }\end{array}$ & $\begin{array}{l}\text { t.valu } \\
\mathrm{e}\end{array}$ & $\begin{array}{l}\text { p.valu } \\
\text { e }\end{array}$ & $\begin{array}{l}\text { Remar } \\
\text { ks }\end{array}$ & $\begin{array}{l}\text { Efficacy } \\
\%\end{array}$ \\
\hline \multirow[t]{2}{*}{1.} & \multirow[t]{2}{*}{ Shula } & \multirow[t]{2}{*}{$\begin{array}{c}2.93 \pm 0.1 \\
5\end{array}$} & $\mathrm{AT}_{1}$ & $1.6 \pm 0.19$ & $\begin{array}{l}1 \\
4\end{array}$ & 10.58 & $\begin{array}{c}\mathrm{P}<0.0 \\
1\end{array}$ & H.S & 45.45 \\
\hline & & & $\mathrm{AT}_{2}$ & $\begin{array}{c}0.67 \pm 0.1 \\
6\end{array}$ & $\begin{array}{l}1 \\
4\end{array}$ & 12.47 & $\begin{array}{c}\mathrm{P}<0.0 \\
1\end{array}$ & H.S & 77.27 \\
\hline \multirow[t]{2}{*}{2.} & \multirow[t]{2}{*}{ Stambha } & \multirow[t]{2}{*}{$\begin{array}{c}2.33 \pm 0.1 \\
3\end{array}$} & $\mathrm{AT}_{1}$ & $\begin{array}{c}1.33 \pm 0.1 \\
6\end{array}$ & $\begin{array}{l}1 \\
4\end{array}$ & 10.25 & $\begin{array}{c}\mathrm{P}<0.0 \\
1\end{array}$ & H.S & 42.86 \\
\hline & & & $\mathrm{AT}_{2}$ & $\begin{array}{c}0.47 \pm 0.1 \\
3\end{array}$ & $\begin{array}{l}1 \\
4\end{array}$ & 20.55 & $\begin{array}{c}\mathrm{P}<0.0 \\
1\end{array}$ & H.S & 80 \\
\hline \multirow[t]{2}{*}{3.} & \multirow[t]{2}{*}{ Graha } & \multirow[t]{2}{*}{$1.6 \pm 0.13$} & $\mathrm{AT}_{1}$ & $\begin{array}{c}0.87 \pm 0.1 \\
7\end{array}$ & $\begin{array}{l}1 \\
4\end{array}$ & 6.21 & $\begin{array}{c}\mathrm{P}<0.0 \\
1\end{array}$ & H.S & 45.83 \\
\hline & & & $\mathrm{AT}_{2}$ & $\begin{array}{c}0.33 \pm 0.1 \\
3\end{array}$ & $\begin{array}{l}1 \\
4\end{array}$ & 10.72 & $\begin{array}{c}\mathrm{P}<0.0 \\
1\end{array}$ & H.S & 79.17 \\
\hline \multirow[t]{2}{*}{4.} & \multirow[t]{2}{*}{ Shotha } & \multirow[t]{2}{*}{$\begin{array}{c}2.27 \pm 0.1 \\
5\end{array}$} & $\mathrm{AT}_{1}$ & $\begin{array}{c}1.33 \pm 0.1 \\
9\end{array}$ & $\begin{array}{l}1 \\
4\end{array}$ & 7.90 & $\begin{array}{c}\mathrm{P}<0.0 \\
1\end{array}$ & H.S & 41.18 \\
\hline & & & $\mathrm{AT}_{2}$ & $\begin{array}{c}0.47 \pm 0.1 \\
3\end{array}$ & $\begin{array}{l}1 \\
4\end{array}$ & 12.44 & $\begin{array}{c}\mathrm{P}<0.0 \\
1\end{array}$ & H.S & 79.41 \\
\hline \multirow[t]{2}{*}{5.} & \multirow[t]{2}{*}{$\begin{array}{l}\text { Vivarnat } \\
\text { a }\end{array}$} & \multirow[t]{2}{*}{$\begin{array}{c}2.07 \pm 0.1 \\
5\end{array}$} & $\mathrm{AT}_{1}$ & $\begin{array}{c}1.47 \pm 0.1 \\
9\end{array}$ & $\begin{array}{l}1 \\
4\end{array}$ & 4.58 & $\begin{array}{c}\mathrm{P}<0.0 \\
1\end{array}$ & H.S & 29.03 \\
\hline & & & $\mathrm{AT}_{2}$ & $\begin{array}{c}0.47 \pm 0.1 \\
3\end{array}$ & $\begin{array}{l}1 \\
4\end{array}$ & 9.80 & $\begin{array}{c}\mathrm{P}<0.0 \\
1\end{array}$ & H.S & 77.42 \\
\hline
\end{tabular}

\section{Group B - Subjective Parameters}

\begin{tabular}{|c|c|c|c|c|c|c|c|c|c|}
\hline $\begin{array}{c}\text { Sr.N } \\
0\end{array}$ & $\begin{array}{c}\text { Sympto } \\
\text { ms }\end{array}$ & $\begin{array}{l}\text { B.T } \\
\text { Mean } \pm S \\
. E\end{array}$ & $\begin{array}{l}\text { Follow } \\
\text { up }\end{array}$ & $\begin{array}{l}\text { A.T } \\
\text { Mean } \pm S \\
. \mathrm{E}\end{array}$ & $\begin{array}{l}\text { d. } \\
\text { f }\end{array}$ & $\begin{array}{l}\text { t.valu } \\
\mathrm{e}^{-}\end{array}$ & $\begin{array}{l}\text { p.valu } \\
\text { e }\end{array}$ & $\begin{array}{l}\text { Remar } \\
\text { ks }\end{array}$ & $\begin{array}{l}\text { Efficacy } \\
\%\end{array}$ \\
\hline \multirow[t]{2}{*}{1.} & \multirow[t]{2}{*}{ Shula } & \multirow[t]{2}{*}{$\begin{array}{c}2.93 \pm 0.1 \\
5\end{array}$} & $\mathrm{AT}_{1}$ & $2.0 \pm 0.20$ & $\begin{array}{l}1 \\
4\end{array}$ & 7.90 & $\begin{array}{c}\mathrm{P}<0.0 \\
1\end{array}$ & H.S & 31.82 \\
\hline & & & $\mathrm{AT}_{2}$ & $\begin{array}{c}1.33 \pm 0.2 \\
5\end{array}$ & $\begin{array}{l}1 \\
4 \\
\end{array}$ & 9.80 & $\begin{array}{c}\mathrm{P}<0.0 \\
1\end{array}$ & H.S & 54.55 \\
\hline \multirow[t]{2}{*}{2.} & \multirow[t]{2}{*}{ Stambha } & \multirow[t]{2}{*}{$\begin{array}{c}2.20 \pm 0.1 \\
5\end{array}$} & $\mathrm{AT}_{1}$ & $\begin{array}{c}1.47 \pm 0.1 \\
9\end{array}$ & $\begin{array}{l}1 \\
4 \\
\end{array}$ & 6.21 & $\begin{array}{c}\mathrm{P}<0.0 \\
1\end{array}$ & H.S & 33.33 \\
\hline & & & $\mathrm{AT}_{2}$ & $\begin{array}{c}1.07 \pm 0.2 \\
3\end{array}$ & $\begin{array}{l}1 \\
4\end{array}$ & 6.85 & $\begin{array}{c}\mathrm{P}<0.0 \\
1\end{array}$ & H.S & 51.52 \\
\hline \multirow[t]{2}{*}{3.} & \multirow[t]{2}{*}{ Graha } & \multirow[t]{2}{*}{$1.6 \pm 0.13$} & $\mathrm{AT}_{1}$ & $\begin{array}{c}1.23 \pm 0.1 \\
5\end{array}$ & $\begin{array}{l}1 \\
4\end{array}$ & 2.65 & $\begin{array}{c}\mathrm{P}<0.0 \\
5\end{array}$ & $S$ & 20.83 \\
\hline & & & $\mathrm{AT}_{2}$ & $\begin{array}{c}0.87 \pm 0.1 \\
9\end{array}$ & $\begin{array}{l}1 \\
4 \\
\end{array}$ & 4.78 & $\begin{array}{c}\mathrm{P}<0.0 \\
1\end{array}$ & H.S & 45.83 \\
\hline
\end{tabular}




\begin{tabular}{|c|c|c|c|c|c|c|c|c|c|}
\hline \multirow[t]{2}{*}{4.} & \multirow[t]{2}{*}{ Shotha } & \multirow[t]{2}{*}{$\begin{array}{c}2.47 \pm 0.1 \\
3\end{array}$} & $\mathrm{AT}_{1}$ & $\begin{array}{c}1.67 \pm 0.1 \\
6\end{array}$ & $\begin{array}{l}1 \\
4\end{array}$ & 7.48 & $\begin{array}{c}\mathrm{P}<0.0 \\
1\end{array}$ & H.S & 32.43 \\
\hline & & & $\mathrm{AT}_{2}$ & $\begin{array}{c}1.07 \pm 0.2 \\
5\end{array}$ & $\begin{array}{l}1 \\
4\end{array}$ & 8.57 & $\begin{array}{c}\mathrm{P}<0.0 \\
1\end{array}$ & H.S & 56.76 \\
\hline \multirow[t]{2}{*}{5.} & \multirow[t]{2}{*}{$\begin{array}{c}\text { Vivarnat } \\
\text { a }\end{array}$} & \multirow[t]{2}{*}{$2.0 \pm 0.17$} & $\mathrm{AT}_{1}$ & $\begin{array}{c}1.60 \pm 0.1 \\
6\end{array}$ & $\begin{array}{l}1 \\
4\end{array}$ & 3.06 & $\begin{array}{c}\mathrm{P}<0.0 \\
1\end{array}$ & H.S & 20 \\
\hline & & & $\mathrm{AT}_{2}$ & $\begin{array}{c}1.13 \pm 0.2 \\
4\end{array}$ & $\begin{array}{l}1 \\
4\end{array}$ & 5.25 & $\begin{array}{c}\mathrm{P}<0.0 \\
1\end{array}$ & H.S & 43.33 \\
\hline
\end{tabular}

Group A - Objective Parameters

\begin{tabular}{|c|c|c|c|c|c|c|c|c|c|}
\hline $\begin{array}{l}\text { Sr.N } \\
\text { o }\end{array}$ & $\begin{array}{c}\text { Paramet } \\
\text { ers }\end{array}$ & $\begin{array}{l}\text { B.T } \\
\text { Mean } \pm \text { S.E }\end{array}$ & $\begin{array}{l}\text { Follow } \\
\text { up }\end{array}$ & $\begin{array}{l}\text { A.T } \\
\text { Mean } \pm \text { S.E }\end{array}$ & $\begin{array}{l}\text { d. } \\
\text { f }\end{array}$ & $\begin{array}{l}\text { t.val } \\
\text { ue }\end{array}$ & $\begin{array}{l}\text { p.val } \\
\text { ue }\end{array}$ & $\begin{array}{l}\text { Remar } \\
\text { ks }\end{array}$ & $\begin{array}{l}\text { Efficac } \\
\text { y\% }\end{array}$ \\
\hline \multirow[t]{2}{*}{1.} & \multirow[t]{2}{*}{ ESR } & \multirow[t]{2}{*}{$\begin{array}{c}53.80 \pm 4.8 \\
6\end{array}$} & $\mathrm{AT}_{1}$ & $\begin{array}{c}45.33 \pm 4.6 \\
9\end{array}$ & $\begin{array}{l}1 \\
4\end{array}$ & 5.13 & $\begin{array}{c}\mathrm{P}<0 . \\
01\end{array}$ & H.S & 15.74 \\
\hline & & & $\mathrm{AT}_{2}$ & $\begin{array}{c}36.00 \pm 3.5 \\
6\end{array}$ & $\begin{array}{l}1 \\
4\end{array}$ & 8.21 & $\begin{array}{c}\mathrm{P}<0 . \\
01\end{array}$ & H.S & 33.09 \\
\hline \multirow[t]{2}{*}{2.} & \multirow[t]{2}{*}{$\begin{array}{l}\text { ASO } \\
\text { Titre }\end{array}$} & \multirow[t]{2}{*}{$\begin{array}{c}305.13 \pm 16 \\
.89\end{array}$} & $\mathrm{AT}_{1}$ & $\begin{array}{c}289.53 \pm 15 \\
.94\end{array}$ & $\begin{array}{l}1 \\
4\end{array}$ & 9.51 & $\begin{array}{c}\mathrm{P}<0 . \\
01\end{array}$ & H.S & 5.11 \\
\hline & & & $\mathrm{AT}_{2}$ & $\begin{array}{c}268.93 \pm 15 \\
.16\end{array}$ & $\begin{array}{l}1 \\
4\end{array}$ & $\begin{array}{c}11.2 \\
9\end{array}$ & $\begin{array}{c}\mathrm{P}<0 . \\
01\end{array}$ & H.S & 11.86 \\
\hline
\end{tabular}

Group B - Objective Parameters

\begin{tabular}{|c|c|c|c|c|c|c|c|c|c|}
\hline $\begin{array}{l}\text { Sr.N } \\
\text { o }\end{array}$ & $\begin{array}{c}\text { Paramet } \\
\text { ers }\end{array}$ & $\begin{array}{l}\text { B.T } \\
\text { Mean } \pm \text { S.E }\end{array}$ & $\begin{array}{l}\text { Follow } \\
\text { up }\end{array}$ & $\begin{array}{l}\text { A.T } \\
\text { Mean } \pm \text { S.E }\end{array}$ & $\begin{array}{l}\text { d. } \\
\text { f }\end{array}$ & $\begin{array}{l}\text { t.val } \\
\text { ue }\end{array}$ & $\begin{array}{l}\text { p.val } \\
\text { ue }\end{array}$ & $\begin{array}{l}\text { Remar } \\
\text { ks }\end{array}$ & $\begin{array}{l}\text { Efficac } \\
\text { y\% }\end{array}$ \\
\hline \multirow[t]{2}{*}{1.} & \multirow[t]{2}{*}{ ESR } & \multirow[t]{2}{*}{$\begin{array}{c}52.53 \pm 4.3 \\
9\end{array}$} & $\mathrm{AT}_{1}$ & $\begin{array}{c}45.87 \pm 4.3 \\
8\end{array}$ & $\begin{array}{l}1 \\
4\end{array}$ & 6.52 & $\begin{array}{c}\mathrm{P}<0 . \\
01\end{array}$ & H.S & 12.69 \\
\hline & & & $\mathrm{AT}_{2}$ & $\begin{array}{c}39.87 \pm 3.6 \\
2\end{array}$ & $\begin{array}{l}1 \\
4\end{array}$ & 6.95 & $\begin{array}{c}\mathrm{P}<0 . \\
01\end{array}$ & H.S & 24.11 \\
\hline \multirow[t]{2}{*}{2.} & \multirow[t]{2}{*}{$\begin{array}{l}\text { ASO } \\
\text { Titre }\end{array}$} & \multirow[t]{2}{*}{$\begin{array}{c}298.67 \pm 14 \\
.14\end{array}$} & $\mathrm{AT}_{1}$ & $\begin{array}{c}284.53 \pm 14 \\
.01\end{array}$ & $\begin{array}{l}1 \\
4\end{array}$ & $\begin{array}{c}10.4 \\
8\end{array}$ & $\begin{array}{c}\mathrm{P}<0 . \\
01\end{array}$ & H.S & 4.73 \\
\hline & & & $\mathrm{AT}_{2}$ & $\begin{array}{c}271.13 \pm 13 \\
.85\end{array}$ & $\begin{array}{l}1 \\
4\end{array}$ & $\begin{array}{c}10.7 \\
9\end{array}$ & $\begin{array}{c}\mathrm{P}<0 . \\
01\end{array}$ & H.S & 9.22 \\
\hline
\end{tabular}

\section{Discussion on Results}

Effect on Sandhishula: - Relief in Sandhishula was observed $77.27 \%$ among the patients of Group-A while the patients of Group-B showed 54.55\% improvement. Both the results were statistically highly significant $(\mathrm{P}<0.01)$.

Effect on Stambha: - $80 \%$ relief was observed in Sandhistambha among the patients of Group-A while the patients of Group-B showed 51.52\% improvement. The relief was statistically highly significant $(\mathrm{P}<0.01)$ in both the groups.
Effect on Graha: - In both the groups, highly significant results were recorded in Graha of the joints. However the percentage was found to be higher in Group- A $79.17 \%$ compared to Group-B $45.83 \%$. Statistically both were highly significant $(\mathrm{P}<0.01)$.

Effect on Sandhishotha: - In both the Groups highly significant $\quad(\mathrm{P}<0.01)$ improvement was recorded in inflammation. Group - A showed 79.41\% relief and Group B showed $56.76 \%$ relief. 
Effect on Vivarnata: - The effect on Vivarnata was observed as $77.42 \%$ in group-A patients while it recorded as $43.33 \%$ in group-B. The improvement was statistically highly significant $(\mathrm{P}<0.01)$.

Effect on ESR: - In Group A, change in ESR value was $33.09 \%$ while in Group B it was $24.11 \%$ in E.S.R. value. The results found in both groups were statistically highly significant $(\mathrm{P}<0.01)$.

Effect on ASO Titre: - In Group A change was $11.86 \%$ while in Group B it was $9.22 \%$ . The results found in both groups were statistically highly significant $(\mathrm{P}<0.01)$.

\section{Overall Effect of Therapy: -}

The overall effect of therapy in Group A was $78.57 \%$ and in Group B was $51.19 \%$.

\section{Conclusion}

Both the Groups show highly significant results but improvement was better in Group A in comparison to Group B. Basti has provided better relief in most of the Cardinal, General and Associated features of the disease at significant level. Comparatively Prasarini taila Basti was found well tolerable in all age groups with minimum complications.

\section{References}

1. Srinivasulu M: Essence of Principles and Practice of Ayurvedic treatment, Varanasi, Chowkambha Publication, Edition 2013, P. No. 372.

2. Ayurvedacharya shree Yadunandanopadyaya:

Madhavanidana with Madhukosha Teeka by Madhavakara, Purvardha, Varanasi, Choukambha Publication, Reprint-2008, P.No. 508.

3. Vaidya Lakshmipati Shastree: Yoga Ratnakara with Hindi Teeka, Purvardha, Varanasi, Choukambha Publication, Reprint-2010, P.No. 566.

4. Bramhananda Tripathi: Sharangadhara Samhita with Deepika Hindi Teeka by Sharangadhara, Madhyama Khanda, Varanasi, Choukambha Publication, Reprint-2010, P.No. 508.

5. Bramhananda Tripathi: Sharangadhara Samhita with Deepika Hindi Teeka by Sharangadhara, Madhyama Khanda, Varanasi, Choukambha Publication, Reprint-2010, P.No. 363.

6. Indradeva Tripathi: Chakradatta with Vaidyaprabha hindi teeka by Chakrapanidatta, Varanasi, Choukambha Publication, Reprint2006, P.No. 455. 